\title{
LA-UR-13-22828
}

Approved for public release; distribution is unlimited.

Title:

Author(s):

Intended for:

Issued:

\section{Annual Report on Institutional Computing Resoures}

\author{
Reisner, Jon M.
}

Report

2013-04-22

\section{- LosAlamos NATIONAL LABORATORY

\author{
EST. 1943
}

Disclaimer:

Los Alamos National Laboratory, an affirmative action/equal opportunity employer,is operated by the Los Alamos National

Security, LLC for the National NuclearSecurity Administration of the U.S. Department of Energy under contract DE-AC52-06NA25396. By approving this article, the publisher recognizes that the U.S. Government retains nonexclusive, royalty-free license to publish or reproduce the published form of this contribution, or to allow others to do so, for U.S. Government purposes.

Los Alamos National Laboratory requests that the publisher identify this article as work performed under the auspices of the

U.S. Departmentof Energy. Los Alamos National Laboratory strongly supports academic freedom and a researcher's right to publish; as an institution, however, the Laboratory does not endorse the viewpoint of a publication or guarantee its technical correctness. 


\title{
Multi-scale Modeling of Green House Gas Emissions Performed on Institutional Computing Project HIGRADchem
}

\author{
Keeley R. Costigan ${ }^{1}$, Sang-Hyun Lee ${ }^{1}$, Jon Reisner ${ }^{1}$ \\ ${ }^{1}$ Los Alamos National Laboratory \\ ${ }^{2}$ New Mexico Consortium
}

Agreements to limit greenhouse gas emissions require scientifically valid methods for monitoring and validating anthropogenic emissions. The task of monitoring $\mathrm{CO}_{2}$ emissions is difficult because relatively small increases need to be detected against $\mathrm{CO}_{2}$ 's variable and large background concentrations. To ensure fair compliance everywhere on the globe, remotely sensed measurements and an understanding of the atmospheric transport of $\mathrm{CO}_{2}$ from its sources are required. The climate treaty verification LDRD project addresses these challenges. The Remote Sensing Verification Project (RSVP) test-bed located in the Four Corners region of Arizona, Utah, Colorado, and New Mexico offers a unique opportunity to develop new approaches for estimating emissions of $\mathrm{CO}_{2}$. Two major power plants located in this area produce very large signals of co-emitted $\mathrm{CO}_{2}$ and $\mathrm{NO}_{2}$ in this rural region. In addition to the Environmental Protection Agency (EPA) maintaining Continuous Emissions Monitoring Systems (CEMS) on each of the power plant stacks, the RSVP program has deployed an array of in-situ and remote sensing instruments, which provide both point and integrated measurements.

To aid in the synthesis and interpretation of the measurements, a multi-scale atmospheric modeling approach is implemented, using two atmospheric numerical models: the Weather Research and Forecasting Model with chemistry (WRF-Chem; Grell et al., 2005) and the HIGRAD model (Reisner et al., 2003). The high fidelity HIGRAD model incorporates a multi-phase Lagrangian particle based approach to track individual chemical species of stack plumes at ultra-high resolution, using an adaptive mesh. It is particularly suited to model buoyancy effects and entrainment processes at the edges of the power plant plumes. WRF-Chem is a community model that has been applied to a number of air quality problems and offers several physical and chemical schemes that can be used to model the transport and chemical transformation of the anthropogenic plumes out of the local region. Multiple nested grids employed in this study allow the model to incorporate atmospheric variability ranging from synoptic scales to micro-scales $(\sim 200 \mathrm{~m})$, while including locally developed flows influenced by the nearby complex terrain of the San Juan Mountains.

An initial test case of 15 March was chosen for simulation. On 15 March 2011, the Four Corners region experienced sunny conditions, under the influence of synopticscale high pressure. Time series of remotely sensed and in-situ $\mathrm{CO}_{2}$ concentrations both showed peaks that suggested the power plant plumes were directed toward the RSVP site. For this case, WRF-Chem employed five nested grids, with horizontal grid spacing from $16.2 \mathrm{~km}$ to $200 \mathrm{~m}$. U.S. Environmental Protection Agency (EPA) National Emissions Inventory (NEI) 2005 inventory and Vulcan (Vulcan, 2009) provide regional emissions in the three largest grids and hourly CEMS 
measurements are used for emissions from the power plants. These simulations agree reasonably well with wind measurements at the RSVP plant and support the theory that the peak concentrations occur when the San Juan plume passes over the site. The simulations also give similar $\mathrm{NO}_{2}$ concentrations to satellite measurements.

The Four Corners simulations with WRF-Chem have expanded over the last year. Additional simulations of 15 Mar 2011 power plant plumes have included modification of the model initial background concentration profiles to provide more realistic chemical initial conditions and observational nudging of the meteorological fields to improve agreement with ground measurements of in-situ and column $\mathrm{CO}_{2}$ concentrations. More case studies of days with observations of interest include 6 June 2012. We are using the model predicted vertical profiles of $\mathrm{CO}_{2}$ for comparison to and analysis of a groundbreaking technique to derive vertical profiles of $\mathrm{CO}_{2}$ from the measurements made at Four Corners.

Our WRF-Chem simulations have also been applied to study broad area methane sources in the Four Corners region and point methane source in the Rocky Mountain Oilfield Test Center (RMOTC) in Wyoming. The preliminary Four-Corners simulation (also of 15 Mar 2011) is part of a study to understand the observed high methane measurement from the RSVP site and observed by satellite. This work has facilitated collaboration with NASA's Jet Propulsion Laboratory (JPL) and is continuing into the IC project extension, where global methane emissions inventories will be used. The initial RMOTC simulation of 26 June 2012, is being used for planning a field experiment for an addition joint project with JPL and Chevron.

We are also running new simulations of $\mathrm{CO}_{2}$ emissions and transport in the Los Angeles basin with updated area emissions and WRF-Chem model code. The Los Angeles simulations employ four nested grids and are run for 24-28 March 2008, when column measurements indicate the influence of weather conditions on measured concentrations. These results are being prepared for publication.

HIGRAD ensemble-based simulations that resolve the momentum, energy, and mass from the four-corner power plant were also conducted during the past year. The simulations use very high resolution near the stack, $2 \mathrm{~m}$, to resolve the flow field produced by the power plant. The primary goal of the ensemble calculations are to estimate the amount of $\mathrm{CO}_{2}$ that is leaving the stack given uncertainty in upstream wind conditions and the combustion process that can lead to large differences in the amount of mass leaving the stacks.

As part of Dubey's LDRD-DR project, a new instrument was fielded to remotely observe $\mathrm{CO}_{2}$ within a column of air. Given this observation, possibly averaged in time, and the HIGRAD ensemble calculations a variety of different inverse approaches are being examined with regard to their ability to estimate the $\mathrm{CO}_{2}$ source. Further, the HIGRAD ensemble calculations are unique in that both Lagrangian and Eulerian calculations are being undertaken to examine the impact of numerical errors on this inverse process (see Figs. 1-3). The results from these 
ensemble calculations and inverse procedures are currently being written up into a paper (see below).

Given the success of this LDRD-DR project, partially made possible by the IC computer allocation, Dubey is pursuing a similar funded project with Chevron to examine the amount of methane that escapes during the fracking process.

\section{References:}

- Grell, G.A., S.E. Peckham, R. Schmitz, S.A. McKeen, G. Frost, W.C. Skamarock and B. Eder, 2005: Fully coupled online chemistry within the WRF model. Atmos. Environ., 39, 6957-6975.

- Reisner, J., A. Wyszogrodzki, V. Mousseau, and D. Knoll, 2003: An efficient physicsbased preconditioner of the fully implicit solution of small-scale thermally driven atmospheric flows. J Comput. Physics., 189, 30-44.

-Vulcan Project, Gurney, K.R., D. Mendoza, Y. Zhou, M Fischer, C. Miller, S. Geethakumar, S. de la Rue du Can (2009) The Vulcan Project: High resolution fossil fuel combustion $\mathrm{CO}_{2}$ emissions fluxes for the United States, Environ. Sci. Technol, 43, doi:10.1021/es900,806c.

\section{Publications:}

Costigan, K., S.-H. Lee, J. Reisner, M. Dubey, S. Love, B. Henderson, and P. Chylek, 2011: Multi-scale Modeling of Power Plant Plume Emissions and Comparisons with Observations. Poster Presentation American Geophysical Union Fall Meeting 2011. San Francisco, CA 4-9 Dec 2011. Presentation number A41B-0085. LA-UR 1112139.

\section{Papers in preparation:}

Paper in preparation on the WRF-Chem simulations of Los Angeles urban $\mathrm{CO}_{2}$ by K.R. Costigan.

Paper in preparation on the multi-scale modeling of power plant chemical plumes using a one-way nested large-eddy simulation for interpretation of ground-based remote-sensing measurements by S.-H. Lee, K. R. Costigan, and M. K. Dubey

Paper in preparation on the vertical profiles obtained from the measurements by $\mathrm{R}$. Lindenmaier, M. Dubey, K.R. Costigan, and S.-H. Lee.

Paper in preparation on inverse modeling approaches applied to the four-corner power plant by M. Pratola, J. Reisner, and H. Godinez. 

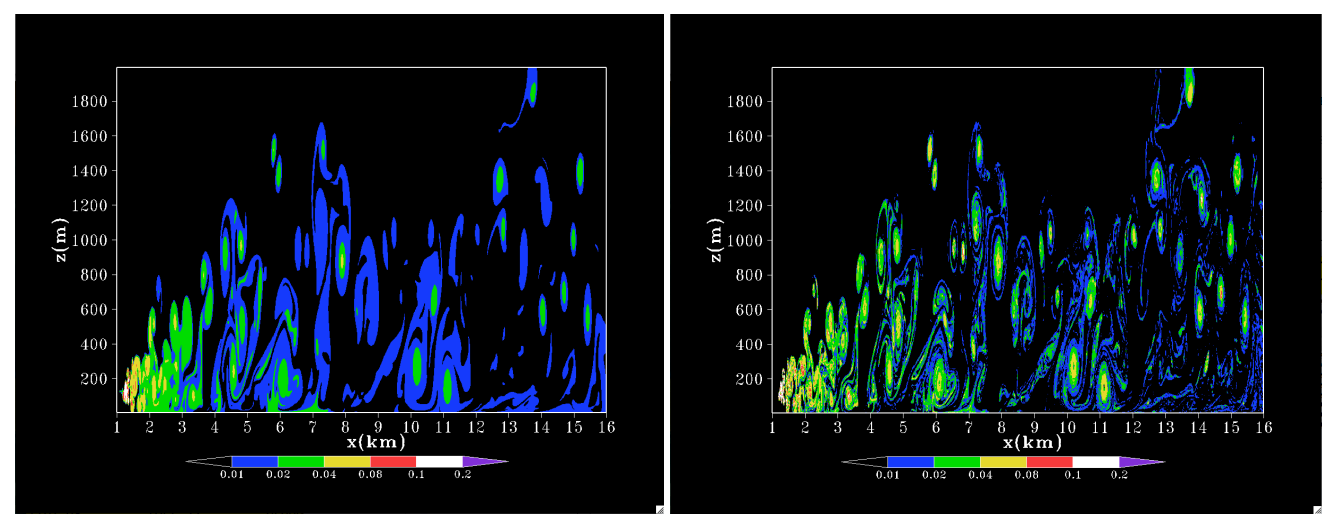

Fig 1: A Eulerian member (left panel) and a Lagrangian member (right panel) of a vertical cross-section of $\mathrm{CO}_{2}$ at the end of the simulation from a HIGRAD ensemble calculation used to estimate the $\mathrm{CO}_{2}$ source from the fourcorner power plant. The Eulerian calculation shows clear evidence of numerical diffusion that may impact the ability of the inverse calculation to estimate the source.
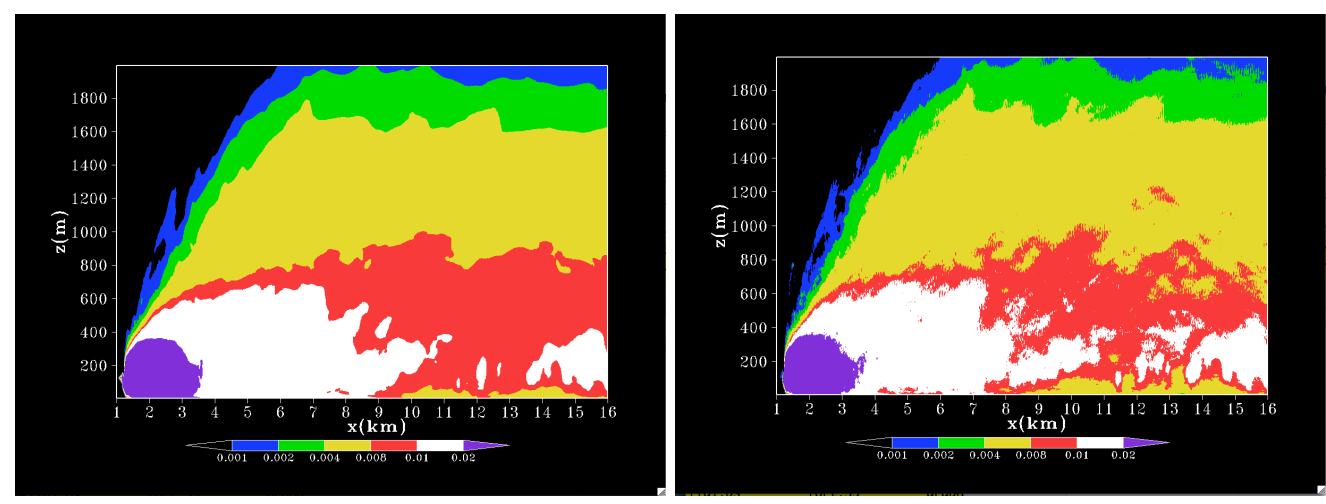

Fig 2: A time-averaged Eulerian member (left panel) and a time-averaged Lagrangian member (right panel) of a vertical cross-section of $\mathrm{CO}_{2}$ from a HIGRAD ensemble calculation used to estimate the $\mathrm{CO}_{2}$ source from the fourcorner power plant. Temporal averaging leads to much smoother fields of $\mathrm{CO}_{2}$ and better agreement between the two numerical approaches. And, if temporal averaged observational data of $\mathrm{CO}_{2}$ can be obtained, highly reliable source estimates can be achieved. 

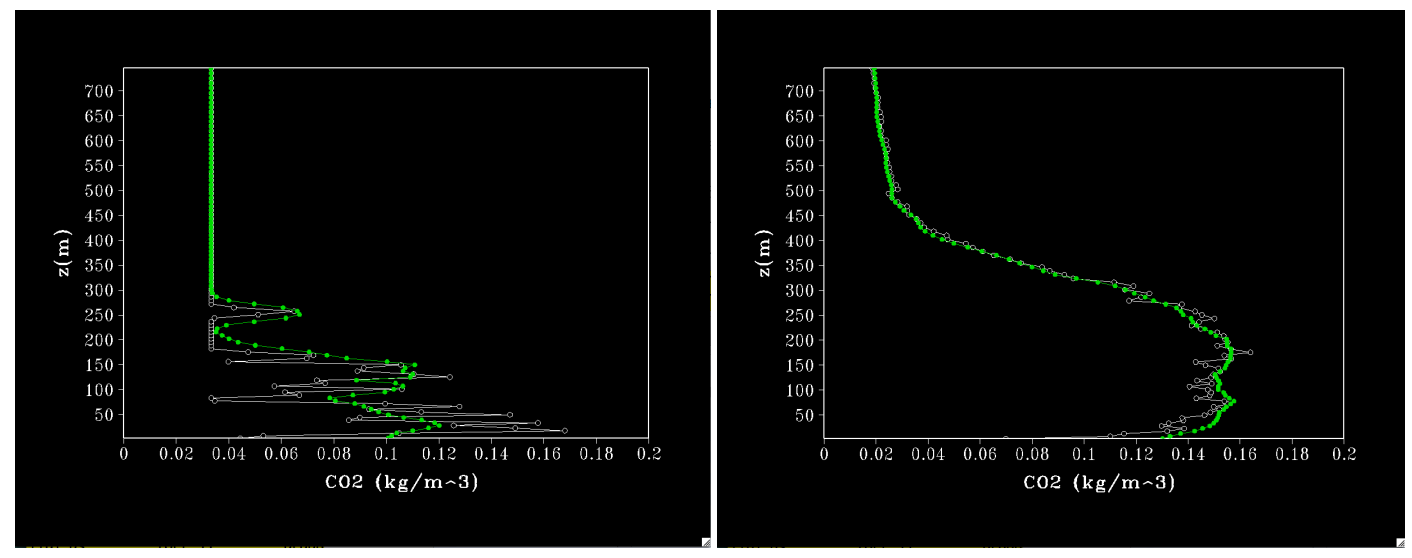

Fig. 3: Vertical profiles of $\mathrm{CO}_{2} 0.5 \mathrm{~km}$ downstream of the $\mathrm{CO}_{2}$ sources shown in Figs 1-2. The green lines are from the Eulerian calculations, whereas the white lines are from the Lagrangian simulations. The left panel shows results at the end of the simulation and the right panel shows results that have been averaged in time. The two panels show that when results are averaged in time, the large differences in $\mathrm{CO}_{2}$ produced by the two disparate numerical schemes start to disappear. 